\title{
Interacting particles system revisited in the framework of the $q$-deformed algebra
}

\author{
A.M. Scarfone ${ }^{1}$, and P. Narayana Swamy ${ }^{2}$ \\ ${ }^{1}$ Dipartimento di Fisica and Istituto Nazionale per la Fisica della Materia \\ (CNR-INFM), Sezione del Politecnico di Torino, I-10129, Italy \\ ${ }^{2}$ Department of Physics, Southern Illinois University, Edwardsville, IL 62026, U.S.A.
}

\begin{abstract}
We discuss the possibility of interpreting a $q$-deformed non-interacting system as incorporating the effects of interactions among its particles. This can be accomplished, for instance, in an ensemble of $q$-Bosons by means of the virial expansion of a real gas in powers of the deformed parameter. The lowest order virial coefficient reduces to the case of the standard, non-interacting Bose gas, while the higher order virial coefficients contain effects arising from the interaction. The same picture can be drawn in a quantum mechanical system where it is shown that the $q$-deformed momentum can be expanded in a series contains high-order powers of the standard quantum phase-space variables. Motivated by this result, we introduce, in the classical framework, a transformation relating the momentum of a free system with the momentum of an interacting system. It is shown that the canonical quantization applied to the interacting system imply a $q$-deformed quantization for the free system.
\end{abstract}

Submitted to: $J P A$

PACS numbers: 05.30.-d, 02.20.Uw, 05.70.Ce, 05.90.+m

E-mail: antonio.scarfone@polito.it, pswamy@siue.edu 


\section{Introduction}

The earliest studies to develop a new realization of the quantum group $\mathrm{SU}_{q}(2)$ are perhaps those of Biedenharn and Mcfarlane [1, 2]. This construction, which is now a standard procedure in the literature, is based on the following algebra describing a $q$-Bosons oscillators system

$$
\begin{aligned}
& \tilde{a} \tilde{a}^{\dagger}-q \tilde{a}^{\dagger} \tilde{a}=1, \quad[\tilde{a}, \tilde{a}]=0, \quad\left[\tilde{a}^{\dagger}, \tilde{a}^{\dagger}\right]=0, \\
& {[N, \tilde{a}]=-\tilde{a}, \quad\left[N, \tilde{a}^{\dagger}\right]=\tilde{a}^{\dagger},}
\end{aligned}
$$

where $q$ is a real deformation parameter. The $q$-Boson Hamiltonian operator is $H=\frac{1}{2} \omega\left(\tilde{a}^{\dagger} \tilde{a}+\tilde{a} \tilde{a}^{\dagger}\right)$ (hereinafter we use unities with $\hbar=1$ ), where eigenvalues $E_{n}=$ $\frac{1}{2} \omega([n+1]+[n])$ are expressed in terms of the basic numbers $[n]=\left(q^{n}-1\right) /(q-1)$ and the Fock space of the $q$-Boson states is constructed according to $|n\rangle=(1 / \sqrt{[n] !})\left(a^{\dagger}\right)^{n}|0\rangle$, where the $q$-factorial is given by $[n] !=[n][n-1] \ldots[1]$, when non-zero.

Starting from the above formalism, the statistical mechanics of a $q$-Bosons gas has been investigated rather extensively in literature [3, 4, 5, 6, 7]. Recent studies on the nature of the thermodynamics as well as properties relating to the algebra and Heisenberg's equation of motion have been worthwhile [8]. More recently, specific topics relating to the thermostatistics of $q$-deformed Boltzmann, Bose and Fermi systems have been investigated in detail [9, 10]. These studies, have not only established detailed results for the thermostatistical properties of ideal $q$-Bosons and $q$-Fermions systems, but they have also illustrated how the deformation of classical systems can be described by means of the $q$-exponential functions and the concomitant Fokker-Planck equation [11. In addition, the study of $q$-Bosons and $q$-Fermions is particularly instructive since it has been shown that the $q$-calculus based on the Jackson derivative can be successfully used to modify standard thermodynamic relations in such a way that the theory of these ideal $q$-deformed systems can be formulated in a self-consistent manner.

Moreover, classical systems described by the $q$-deformed Poisson bracket has been investigated [12] in order to understand the origin of the deformed structure from the underlying dynamics governing some complex systems.

Having accomplished a great deal of understanding of the $q$-deformed systems revealed by recent investigations, we may ask the question whether there are simple physical descriptions of what constitutes a deformation. In other words, it is worthwhile to ask if there are simple manifestations of $q$-deformation and what are the physical basis of such manifestations. It has been observed in [13] that interacting Bosons gas can be described by a Hamiltonian containing various powers of the operators $a$ and $a^{\dagger}$, where the lowest order non-interacting Hamiltonian contains only one pair of these operators. Furthermore, we can learn from a study of non-ideal gases, (see, for instance, [14]), that the state equations of interacting particle systems are well described by means of the virial expansion in terms of higher orders by powers of the number of particles, where the Van der Walls approximations is given by the first two terms in the series.

Therefore, the power series expansion is a convenient tool to describe interacting 
systems, with the lowest order representing the system of non-interacting particles in the ensemble. Unfortunately, very few authors have examined this issue. An exception is given by Parthasarathy and Viswanathan [15] who have developed an interesting interpretation of the origin of the $q$-deformation in an interacting particles system. In fact, it is shown that many thermodynamic quantities of a $q$-Bosons system can be expressed as a power series in powers of $N$ or in series of the deformed parameter $\epsilon=q-1$, implying that the $q$-deformation arises from the interaction among the particles of the ensemble. More precisely, while the zero-th order describes the undeformed (non-interacting) system, the higher order terms contain various powers of $\epsilon$ which are equivalent to different order contributions arising from interactions.

Similar considerations have been presented also in [16], where the perturbative aspects of the $q$-deformed Schrödinger equation are analyzed by means of a high order momentum-dependent interaction originating from the underlying $q$-deformation of the Heisenberg algebra.

In this paper, we shall highlight some aspects concerning the possible interpretation of a $q$-deformed non-interacting system describing a non-deformed interacting system.

The plan of the paper is as follows. In Section II, we present some results to illustrate the idea that $q$-deformation can originate from the presence of an interaction

in the system. This is better clarified in Section III, where we study an interacting Bosons system by means of the virial expansion of a free $q$-Bosons system. In Section IV, we illustrate the same idea for a quantum mechanical system, by revisiting the $q$ deformed Heisenberg algebra. We write down the q-deformed momentum in a series of the standard quantum space-phase variables where the high-order contributions originate from the presence of the interaction into the system. The equivalence of $q$-deformation and interaction for a mechanical system is discussed in the Section $\mathrm{V}$ by introducing a contact transformation which relates the classical counterpart of the canonical quantum momentum (describing an interacting system) to the classical counterpart of the deformed quantum momentum (describing a free system). As a consequence, it is shown that the canonical quantization ( $\grave{a}$ la Dirac) applied to the interacting system implies a $q$-deformed quantization of the free system. Section VI contain a conclusive discussion.

\section{Preliminary results}

We consider a system of interacting particles described by the $q$-oscillator algebra, expressed for a one level system through equation (1.1), where $q$ implements the deformation of the system. The theory of such $q$-Bosons introduces the basic number according to

$$
[N]=\frac{q^{N}-1}{q-1},
$$

where $N$ is the number operator, in order to describe the Fock space of the particles. Hereinafter, we take $q>1$ without loss of generality and we set $\epsilon=q-1$, with $\epsilon \ll 1$. 
Thus, the expression of the basic number is written as

$$
[N]=\epsilon^{-1}\left[(1+\epsilon)^{N}-1\right],
$$

which can be expanded in a series of powers of $N$ according to

$$
\begin{aligned}
{[N]=} & \left(1-\frac{\epsilon}{2}+\frac{\epsilon^{2}}{3}-\frac{\epsilon^{3}}{4}+\ldots\right) N+\frac{\epsilon}{2 !}\left(1-\epsilon+\frac{11}{12} \epsilon^{2}-\ldots\right) N^{2} \\
& +\frac{\epsilon^{2}}{3 !}\left(1-\frac{3}{2} \epsilon+\ldots\right) N^{3}+O\left(N^{4}\right) .
\end{aligned}
$$

This result, in agreement with the interpretation advanced in [15], shows that the quantity $[N]$ incorporates thermal averages of $N, N^{2}, N^{3}$, etc. Further inspection reveals that the contributions from interaction can be viewed either in terms of $N, N^{2}, N^{3}$, etc. or in terms of $\epsilon, \epsilon^{2}, \epsilon^{3}$, etc. In fact, by interpreting the contributions arising from interactions by means of terms containing higher powers of the deformation parameter $\epsilon$, we can rewrite the series (2.3) in the following form

$$
\begin{aligned}
{[N] } & =\sum_{i=0}^{\infty} \frac{\epsilon^{i}}{(i+1) !} \prod_{j=0}^{i}(N-j) \\
& =N+\frac{\epsilon}{2 !} N(N-1)+\frac{\epsilon^{2}}{3 !} N(N-1)(N-2)+O\left(\epsilon^{3}\right),
\end{aligned}
$$

so that, we may regard the above series as describing the interaction among the particles of the system: the $\epsilon \rightarrow 0$ limit describes the non-interacting particles corresponding to an undeformed gas while the higher order terms describe the various orders of interaction. In this way, we can interpret the system of interacting particles as a $q$-deformed free gas.

Next, we consider the standard form of the Hamiltonian for the $q$-Bosons

$$
H_{\epsilon}=\frac{1}{2} \omega([N+1]+[N]),
$$

reducing to the usual Hamiltonian $H_{0}=\omega(N+1 / 2)$ when $\epsilon$ goes to zero. This Hamiltonian follows directly from the $q$-deformed oscillator algebra, with the standard representation $[N]=a^{\dagger} a$ and $[N+1]=a a^{\dagger}$.

The alternative form $H_{\epsilon}=\omega([N]+1 / 2)$ is also commonly used [17], but that is certainly an approximation [18].

Hamiltonian (2.5) can be expressed as

$$
\begin{aligned}
H_{\epsilon}= & \omega\left[\frac{1}{2}+\left(1+\frac{\epsilon^{2}}{12}-\frac{\epsilon^{3}}{12}+\ldots\right) N+\frac{\epsilon}{2}\left(1-\frac{\epsilon}{2}+\frac{5}{12} \epsilon^{2}-\ldots\right) N^{2}\right. \\
& \left.+\frac{\epsilon^{2}}{6}(1-\epsilon+\ldots) N^{3}\right]+O\left(N^{4}\right),
\end{aligned}
$$

which can be rearranged in a series containing higher powers of the deformation parameter according to

$$
H_{\epsilon}=H_{0}+\omega \sum_{i=1}^{\infty} \epsilon^{i} \frac{(2 N+1-i)}{2(i+1) !} \prod_{j=0}^{i-1}(N-j) .
$$


The first term $H_{0}$, surviving in the limit $\epsilon \rightarrow 0$, is the undeformed Hamiltonian corresponding to the non-interacting case, whilst the higher order terms are interpreted as arising from interaction.

Finally, for completeness, let us consider the following transformation

$$
\tilde{a}=\hat{a} \sqrt{\frac{[N]}{N}}
$$

and similarly for $\tilde{a}^{\dagger}$, where $\hat{a}$ and $\hat{a}^{\dagger}$ are the undeformed generators obeying to the ordinary $\mathrm{SU}(2)$ algebra. As is well-known [19, 20], this transformation maps the $q$-algebra (1.1) onto the standard algebra of a non interacting Bosons system. By expanding equation (2.8) in power series, thus one obtains

$$
\begin{aligned}
\tilde{a}=\hat{a}[1 & +\frac{\epsilon}{4}(N-1)-\frac{\epsilon^{2}}{96}(N-1)(5 N+3) \\
& \left.+\frac{\epsilon^{3}}{384}(N-1)(N-5)(3 N-7)+O\left(\epsilon^{4}\right)\right],
\end{aligned}
$$

which shows the equivalence of the two operators $\tilde{a}$ and $\hat{a}$ at the zeroth order. Again, the high order terms can be interpreted as a correction to $\hat{a}$ arising from the presence of the interactions in the system of non-interacting Bosons.

\section{The virial expansion in the $q$-Bosons system}

A good illustration of the idea that a $q$-deformed non-interacting many-body system can be interpreted as describing an non-deformed interacting system can be provided by studying the virial expansion of a $q$-Bosons ensemble although this idea can also be investigated starting from other physically relevant quantities. For instance, one could consider the probability distribution function for a $q$-Bosons system, derived in [10], as

$$
n=\frac{1}{\ln (1-\epsilon)} \ln \left(\frac{y}{y-\epsilon}\right),
$$

where $y=z^{-1} e^{\beta E}-1$ and $z=e^{\beta \mu}$ is the fugacity of the gas. By expanding this quantity in a power series, one obtains

$$
\begin{aligned}
n=\frac{1}{y} & +\frac{\epsilon}{2 y}\left(1+\frac{1}{y}\right)-\frac{\epsilon^{2}}{12 y}\left(1+\frac{1}{y}\right)\left(1-\frac{4}{y}\right) \\
& +\frac{\epsilon^{3}}{24 y}\left(1+\frac{1}{y}\right)\left(1-\frac{2}{y}+\frac{6}{y^{2}}\right)+O\left(\epsilon^{4}\right),
\end{aligned}
$$

where the first term corresponds to the mean occupation number of the standard Bose statistics, whilst the higher order terms represent interaction contributions. We may thus interpret the above as the average occupation number of an interacting ensemble of Bosons.

Most results derived in a thermostatisics theory based on the $q$-calculus involve generalized functions such as $\tilde{g}_{3 / 2}(z, \epsilon)$ or $\tilde{g}_{5 / 2}(z, \epsilon)$. These functions are generalizations of the well-known functions $g_{3 / 2}(z)$ or $g_{5 / 2}(z)$ arising in the standard thermostatistics of a Bosons gas and can be taken as typical of what comes out in the deformed theory 
which we are now interpreting as consequence of the interaction among the many-body system.

We start from the following result derived in [10]

$$
\tilde{g}_{3 / 2}(z, \epsilon)=\frac{\lambda^{3}}{v}
$$

where $v=V / N$ is the specific volume, $\lambda=h /(2 \pi m k T)^{\frac{1}{2}}$ is the thermal wavelength and

$$
\tilde{g}_{3 / 2}(z, \epsilon)=\frac{1}{\ln (1+\epsilon)} \sum_{k=1}^{\infty} \frac{z^{k}}{k^{5 / 2}}\left[(1+\epsilon)^{k}-1\right] .
$$

Expanding this function in a power series, we obtain the result

$$
\begin{aligned}
\tilde{g}_{3 / 2}(z, \epsilon) & =z\left(1+\frac{z}{2 \sqrt{2}}+\frac{z^{2}}{3 \sqrt{3}}+\ldots\right)+\frac{z}{2}\left(1+\frac{z}{\sqrt{2}}+\frac{z^{2}}{\sqrt{3}}+\ldots\right) \epsilon \\
& -\frac{z}{12}\left(1-\frac{z}{\sqrt{2}}-\sqrt{3} z^{2}+\ldots\right) \epsilon^{2}+\frac{z}{24}\left(1+\frac{z^{2}}{\sqrt{3}}+\ldots\right) \epsilon^{3}+O\left(\epsilon^{4}\right) .
\end{aligned}
$$

Note that the zeroth order term is the familiar $g_{3 / 2}(z)$ function encountered in standard Bose statistics [21] whilst the higher order terms can be interpreted as describing contributions from interactions.

In a similar way, we can consider the equation of state for a $q$-Bosons system [10]

$$
\tilde{g}_{5 / 2}(z, \epsilon)=\frac{\lambda^{3} P}{k T},
$$

where the function

$$
\tilde{g}_{5 / 2}(z, \epsilon)=\frac{1}{\ln (1+\epsilon)} \sum_{k=1}^{\infty} \frac{z^{k}}{k^{7 / 2}}\left[(1+\epsilon)^{k}-1\right],
$$

has the following power series expansion

$$
\begin{aligned}
\tilde{g}_{5 / 2}(z, \epsilon) & =z\left(1+\frac{z}{4 \sqrt{2}}+\frac{z^{2}}{9 \sqrt{3}}+\ldots\right)+\frac{z}{2}\left(1+\frac{z}{2 \sqrt{2}}+\frac{z^{2}}{3 \sqrt{3}}+\ldots\right) \epsilon \\
& -\frac{z}{12}\left(1-\frac{z^{2}}{2 \sqrt{2}}-\frac{z^{3}}{\sqrt{3}}+\ldots\right) \epsilon^{2}+\frac{z}{24}\left(1+\frac{z^{2}}{3 \sqrt{3}}+\ldots\right) \epsilon^{3}+O\left(\epsilon^{4}\right) .
\end{aligned}
$$

Again, the zeroth order term coincides with the known function $g_{5 / 2}(z)$ of the standard statistical mechanics, whilst the higher order terms can be interpreted as describing contributions from interactions.

From the results (3.5) and (3.8) we can obtain the following virial expansion

$$
\frac{P v}{k T}=\sum_{k=1}^{\infty} a_{k}(\epsilon)\left(\frac{\lambda^{3}}{v}\right)^{k-1},
$$

where the virial coefficients $a_{k}(\epsilon)$ describe the equation of state of an interacting Bosons system or, equivalently, they describe the equation of state of a $q$-deformed system of 
non-interacting Bosons. The first few terms are

$$
\begin{aligned}
a_{1}(\epsilon) & =1 \\
a_{2}(\epsilon) & =-\frac{1}{4 \sqrt{2}}-\frac{1}{48 \sqrt{2}} \epsilon^{2}(1-\epsilon)+O\left(\epsilon^{4}\right) \\
& =-0.17678-0.01473 \epsilon^{2}+0.01473 \epsilon^{3}+O\left(\epsilon^{4}\right) \\
a_{3}(\epsilon) & =-\left(\frac{2}{9 \sqrt{3}}-\frac{1}{8}\right)-\left(\frac{1}{18 \sqrt{3}}-\frac{1}{48}\right) \epsilon^{2}(1-\epsilon)+O\left(\epsilon^{4}\right) \\
& =-0.00330-0.01124 \epsilon^{2}+0.01124 \epsilon^{3}+O\left(\epsilon^{4}\right) \\
a_{4}(\epsilon) & =-\left(\frac{3}{32}+\frac{5}{32 \sqrt{2}}-\frac{1}{2 \sqrt{6}}\right)-\left(\frac{3}{64}+\frac{5}{128 \sqrt{2}}-\frac{1}{6 \sqrt{6}}\right) \epsilon^{2}(1-\epsilon)+O\left(\epsilon^{4}\right) \\
& =-0.00011-0.00645 \epsilon^{2}+0.00645 \epsilon^{3}+O\left(\epsilon^{4}\right) .
\end{aligned}
$$

(We may note that the first coefficient is exact since it does not contain any corrections).

It is readily observed that when $\epsilon \rightarrow 0$ we recover the virial coefficients of the ordinary (undeformed) non-interacting Bosons system, as shown in standard textbooks [21. The higher order terms arise from the deformation, i.e. we may regard the deviations from the values of the ordinary Bosons system as indicating the presence of suck an interaction among the particles of the system.

\section{Heisenberg algebra and $q$-deformed momentum}

We may further develop the idea of the equivalence among deformation and interaction by considering a quantum mechanical system.

As is known, a (non-Hermitian realization) of algebra (1.1) is provided by means of the $q$-deformed Bargmann-Wigner holomorphism, given by $\tilde{a} \equiv x$ and $\tilde{a}^{\dagger} \equiv D_{x}$, where $D_{x}$ is the Jackson derivative [22], defined in

$$
D_{x}=\frac{1}{x} \frac{\mathcal{D}_{q}-1}{q-1},
$$

with

$$
\mathcal{D}_{q}=q^{x \partial_{x}},
$$

the dilatation operator

$$
\mathcal{D}_{q} f(x)=f(q x) \mathcal{D}_{q} .
$$

According to the $q$-algebra (1.1), by taking into account the relation $[N+1]=q[N]+1$, we can derive the following solution [23, 24]

$$
[N]=x D_{x},
$$

so that the $q$-Boson number operator can be defined as $N=x \partial_{x}$ [see equation (4.1)].

With the purpose of consistently introducing a quantum mechanics theory based on the $q$-calculus, it has been suggested by several authors (see for instance [25, 26, 27]), to replace the quantum momentum operator $\bar{p}=-i \partial_{x}$ with a $q$-deformed version given 
by $\tilde{P}=-i D_{\tilde{X}}$.

In this way, the algebra (1.1) can be written formally in the form

$$
q \tilde{X} \tilde{P}-\tilde{P} \tilde{X}=i,
$$

with $\tilde{X} \equiv x$ the position operators, which reduces to the standard Heisenberg algebra in the $q \rightarrow 1$ limit.

Despite this nice result a certain prudence is indeed required. In fact, while $\bar{p}$ is a Hermitian operator and can be identified with a physical observable, the same is not true for the quantity $\tilde{P}$ which is not Hermitian. Thus, a physical observable corresponding to a $q$-deformed momentum $\tilde{p}$ [28, 29] must be introduced according to a certain procedure such that $\tilde{p}=\tilde{p}^{\dagger}=f\left(\tilde{P}, \tilde{P}^{\dagger}\right)$.

To do this, we first observe that the ordinary commutation among the operators $\tilde{X}$ and $\tilde{P}$ reads

$$
\tilde{X} \tilde{P}-\tilde{P} \tilde{X}=i \mathcal{D}_{q} .
$$

Of course, equations (4.5) and (4.6) represent the same algebra, as can be verified by employing the relation $\mathcal{D}_{q}=1+i(q-1) \tilde{X} \tilde{P}$.

On the other hand, let us consider the Hermitian conjugate of this last equation, given by

$$
\tilde{X} \tilde{P}^{\dagger}-\tilde{P}^{\dagger} \tilde{X}=i\left(q \mathcal{D}_{q}\right)^{-1}
$$

since $\mathcal{D}_{q}^{\dagger}=\left(q \mathcal{D}_{q}\right)^{-1}$ and we assumed $\tilde{X}=\tilde{X}^{\dagger}$. By matching equation (4.5), multiplied on the left hand side by the operator $\left(q \mathcal{D}_{q}\right)^{-1}$ that can be written in

$$
\tilde{X}\left(q \mathcal{D}_{q}\right)^{-1} \tilde{P}-\left(q \mathcal{D}_{q}\right)^{-1} \tilde{P} \tilde{X}=i\left(q \mathcal{D}_{q}\right)^{-1},
$$

with equation (4.7), it is natural to define

$$
\tilde{P}^{\dagger}=\left(q \mathcal{D}_{q}\right)^{-1} \tilde{P}, \quad \text { with } \quad\left(\tilde{P}^{\dagger}\right)^{\dagger} \equiv \tilde{P} .
$$

Accordingly, one introduces the Hermitian operator

$$
\tilde{p}=\frac{1}{2}\left(\tilde{P}+\tilde{P}^{\dagger}\right)
$$

which can be identified with the right $q$-deformed version of the quantum momentum. After rescaling the position operator, according to $\tilde{x}=2 q \tilde{X} /(1+q)$, algebra (4.5) can be finally written in

$$
q^{1 / 2} \tilde{x} \tilde{p}-q^{-1 / 2} \tilde{p} \tilde{x}=i \Lambda_{q}
$$

where the operator $\Lambda_{q}=q^{-1 / 2} \mathcal{D}_{q}^{-1}$ fulfills the further relations

$$
\Lambda_{q} \tilde{x}=q^{-1} \tilde{x} \Lambda_{q}, \quad \Lambda_{q} \tilde{p}=q \tilde{p} \Lambda_{q} .
$$

Equations (4.11)-(4.12) establish the q-deformed Heisenberg's algebra for the phase space quantum variables $\tilde{x}$ and $\tilde{p}$, as known in literature [28, 29].

We can now consider a power series expansion for $\tilde{p}$. To do this, we collect together Eqs. (4.1), (4.9) and (4.10) to obtain

$$
\tilde{p}=-\frac{i}{2 \tilde{X}} \frac{\mathcal{D}_{q}-\mathcal{D}_{q}^{-1}}{q-1} \equiv-\frac{i}{\hat{x}} \frac{q^{i \hat{x} \hat{p}}-q^{-i \hat{x} \hat{p}}}{q-q^{-1}},
$$


with $\hat{x} \equiv \tilde{x}, \hat{p}=-i \partial_{\hat{x}}$, which can be written in the following compact form

$$
\tilde{p}=\hat{p} \frac{[\hat{z}]_{\mathrm{s}}}{\hat{z}},
$$

where $\hat{z}=i \hat{x} \hat{p}$ and $[\hat{z}]_{\mathrm{s}}=\left(q^{\hat{z}}-q^{-\hat{z}}\right) /\left(q-q^{-1}\right)$ is the symmetric version of the basic number. Relation (4.13) is equivalent to the one derived in 28 and successively reconsidered in [16]. It plays the same role as the transformation (2.8) and relates the $q$-deformed operators $\tilde{x}$ and $\tilde{p}$, fulfilling the generalized Heisenberg's algebra (4.11), to the standard operators $\hat{x}$ and $\hat{p}$ which satisfy the undeformed algebra $[\hat{x}, \hat{p}]=i$.

By expanding equation (4.13) in a series of $\epsilon=q-1$, we obtain

$$
\tilde{p}=\hat{p}\left[1-\frac{1}{6}(1+\hat{x} \hat{p} \hat{x} \hat{p}) \epsilon^{2}(1-\epsilon)\right]+O\left(\epsilon^{4}\right),
$$

in accordance with the results reported in [16]. Again, the higher order terms can be interpreted as effects due to interactions, i.e. the first term corresponds to the momentum of an non-interacting system whilst the higher order terms arise from interactions.

\section{The equivalent interaction}

In order to better clarify the question concerning the possible link between interaction and deformation we are asking if there exists a simple physical indication of what constitutes a deformation.

In this respect, we consider a classical system described by the phase-space variables $(X, P)$. For a non-interacting system the energy is only kinetic, i.e. $E=P^{2} /(2 m)$. Afterwards, we introduce a contact transformation which changes the momentum $P \rightarrow p \equiv p(X, P)$ but leave unchanged the coordinate $X \rightarrow x \equiv X$. In the new phase-space variables $(x, p)$, the energy of the system can be written, without loss of generality, in $E=p^{2} /(2 m)+U_{\alpha}(x, p)$ that contains now the extra term $U_{\alpha}(x, p)$ representing an interaction potential, whose explicit form is determined by the contact transformation we are introducing. In particular, we are looking for a transformation able to capture the physical meaning of the $q$-deformation.

On the basis of the results described in the previous section, this can be realized by introducing a transformation such that the quantum analogue of the classical mapping $P \rightarrow p \equiv p(X, P)$ corresponds to the transformation (4.13) among quantum operators. Hereafter, we identify the quantity $\hat{p}$ with the quantum operator of the momentum $p$ (describing the interacting system) and the quantity $\tilde{p}$ with the quantum operator corresponding to the momentum $P$ (describing the free system).

To realize such a result, we introduce the following point transformation

$$
x \rightarrow X \equiv x, \quad p \rightarrow P=\frac{1}{x} \frac{\sin (\alpha x p)}{\sinh (\alpha)},
$$

which imply an interaction term of the kind

$$
U_{\alpha}(x, p)=\frac{\sin ^{2}(\alpha x p)-[\sinh (\alpha) x p]^{2}}{2 m \sinh ^{2}(\alpha) x^{2}},
$$


[derived from the quantity $U_{\alpha}(x, p)=\frac{1}{2 m}\left(P^{2}-p^{2}\right)$ ], where $\alpha=\log q$ is the deformed parameter for the classical system. In the $\alpha \rightarrow 0$ limit equation (5.1) reduces to the identity transformation and the potential $U_{\alpha}(x, p)$ vanishes.

It is easy to verify that, by employing the canonical quantum prescription for the momentum $p$, with $p \rightarrow \hat{p}=-i \partial_{x}$, we obtain a $q$-deformed quantum prescription for the momentum $P$, with $P \rightarrow \tilde{p}$.

In fact, from transformation (5.1), we obtain

$$
P=\frac{\sin (\alpha x p)}{x \sinh (\alpha)} \rightarrow \frac{1}{x} \frac{\sin \left(-i \alpha x \partial_{x}\right)}{\sinh (\alpha)}=-\frac{i}{\hat{x}} \frac{q^{i \hat{x} \hat{p}}-q^{-i \hat{x} \hat{p}}}{q-q^{-1}}=\tilde{p},
$$

in accordance with the transformation (4.13).

We remark that, since transformation (5.1) depends on both the phase-space variables an ordering prescription must be imposed. Consistently with the formalism adopted in the previous section, we employed the standard prescription with the $\hat{x}$ operator on the left and the $\hat{p}$ operator on the right.

An immediate consequence of this argument is that the Schrödinger equation describing a quantum interacting system obtained from the canonical quantization of the classical system governed by the potential $U_{\alpha}(x, p)\left[U_{q}(\hat{x}, \hat{p})=\frac{1}{2 m}\left(\tilde{p}^{2}-\hat{p}^{2}\right)\right.$ in the quantum picture] is transformed in a $q$-deformed Schrödinger equation describing a noninteracting quantum system (and vice versa).

This fact follows readily from the following correspondence

$$
E=\frac{\hat{p}^{2}}{2 m}+U_{q}(\hat{x}, \hat{p}) \quad \Leftrightarrow \quad E=\frac{\tilde{p}^{2}}{2 m} .
$$

Let us point out that the idea to introduce a deformed algebra in order to take into account the effect of interactions is not new at all.

In fact, this is reminiscent of what was done in early quantum mechanics by Bohm and Madelung [30, which recognize that a quantum system, described by the noncommutative operators $\hat{x}$ and $\hat{p}$ acting on the $\psi$-function of a quantum system, behaves like a classical interacting fluid, described by the commutative functions $\rho$ and $S$, whose interaction is described through a suitable quantum potential $U_{\mathrm{BM}}(\rho)$, capable of capturing the quantum feature arising in the Schrödinger picture.

By identifying the quantum momentum $-i \partial_{x} \psi$ in the $\psi$-representation with the canonical momentum $\partial_{x} S$ in the $\rho-S$ representation, the Schrödinger equation can be transformed according to

$$
E=-\frac{1}{2 m} \frac{\partial_{x}^{2} \psi}{\psi} \Rightarrow E=\frac{\left(\partial_{x} S\right)^{2}}{2 m}+U_{\text {Вм }}(\rho),
$$

(endowed by a continuity equation for the field $\rho$ ), where, the transformed equation can be interpreted as a classical Hamilton-Jacobi-like equation for the principal Hamilton function $S$.

In this way, the free system in the Schrödinger picture (in the noncommutative algebraic formalism) is transformed in an interacting classical system (in the commutative 
algebraic formalism) governed by the Bohm-Madelung potential

$$
U_{\mathrm{BM}}(\rho)=-\frac{1}{2 m} \frac{\partial_{x x} \sqrt{\rho}}{\sqrt{\rho}},
$$

that introduces the quantum effects into the system just as the potential (5.2) does with respect to the $q$-deformation.

\section{Conclusion and discussion}

In this work we have investigated a possible interpretation of $q$-deformation in terms of a physical interaction. We analyze several quantities of physical interest such as the Hamiltonian and the occupation number of a $q$-Bosons system showing that all these quantities can be expanded in a powers series of the deformation parameter $\epsilon=q-1$. This leads us to identify the zeroth order quantities corresponding to the description of an undeformed system and the higher order quantities representing the contribution arising from deformation. We can interpret the lowest order quantities characterizing the non-interacting system whilst the higher order contributions arise from the presence of a kind of interaction in the system.

We investigate the virial expansion in the context of the $q$-Bosons system which provides an illustration of the equivalence of the two interpretations. This equivalence arises from the premise that statistical mechanics reveals that a theory of interacting particles, e.g., Bosons, predicts the various virial coefficients so that the equation of state of the gas reflects the presence of interactions in the system. Thus, we have determined explicit forms for the first four virial coefficients which are expressed in a powers series of $\epsilon$ and we have shown explicitly that in the lowest order, these coefficients reduce to those of the standard Bose gas, while the higher order terms describe the effects of the deformation. This idea stems from a study of a non-ideal gas, (e.g. [14]), where the equation of state of interacting systems is described well by the virial expansion in powers of $N$, with the Van der Walls law being just an approximate description provided by the first two terms in the series.

The equivalence between $q$-deformation and interaction has been also illustrated in a quantum mechanical system. The study of the $q$-Heisenberg's algebra enables us to introduce the effective momentum $\tilde{p}$ which can be related by a power series in the ordinary quantum phase-space variables $\hat{x}$ and $\hat{p}$. Based on this result we have considered a point transformation $P \rightarrow p$ for the momentum of a classical system derived by requiring that the canonical quantization rule $p \rightarrow \hat{p}$, for themomentum $p$, imply a $q$-quantization rule $P \rightarrow \tilde{p}$, for the momentum $P$. Accordingly, identifying the $(X, P)$-system with a free system and the $(x, p)$-system with the interacting one we have obtained the expression of the interacting potential $U_{\alpha}(x, p)$ responsible for the $q$-deformation. This means that the classical momentum corresponding to the canonical quantum momentum (describing a suitably interacting system) can be related to the classical momentum corresponding to the deformed quantum momentum (describing a free system) by means of a contact transformation. 
At this stage we are not able to give a physical interpretation, if any, of the potential (5.2). Notwithstanding, it is worth remarking that the idea underling this work, to interpret the deformations of a physics theory as representative of the interactions of the system that the theory is describing, has been also advanced by several authors both for a quantum mechanical system [16] and for a statistical mechanical system [9, 32, 33, 34, 35] and actually is applicable not just to the $q$-formalism employed in this work as a simple illustrative example.

Here, we have used the $q$-deformed algebra, which is well known and largely applied in physics since the beginning of the last century, in order to give an explicit demonstration of how the method should work. There is no physical reason, a priori, to believe that the $q$-deformation is the suitable deformation capable of capturing the physical information contained in any interaction. More appropriately, one should consider a given interacting system and then attempt to realize the corresponding algebraic deformation able to capture the physical feature contained in it. This project however, appears to be beyond our present scope.

\section{References}

[1] Biedenharn L 1989 J. Phys. A: Math. Gen. 22 L873

[2] Mcfarlane A 1989 J. Phys. A: Math. Gen. 224581

[3] Lee C and Yu J 1990 Phys. Lett. A 15063

[4] Yang Q and Xu B 1993 J. Phys. A: Math. Gen. 26 L365

[5] Aizawa N 1993 J. Phys. A: Math. Gen. 261115

[6] Viswanathan K et al 1993 J. Phys. A: Math. Gen. 25 L335

[7] Ramanathan R 1992 Phys. Rev. D 454706

[8] Narayana Swamy P 1996 Int. J. Mod. Phys. B 10 683; 1996 Mod. Phys. Lett. B 10 23; 1998 Int. J. Mod. Phys. B 12 3495; 2006 Int. J. Mod. Phys. B 20697

[9] Lavagno A, Scarfone AM and Narayana Swamy P 2007 J. Phys. A: Math. Theor. 408635

[10] Lavagno A and Narayana Swamy P 2000 Phys. Rev. E 61 1218; 2002 Phys. Rev. E 65036101

[11] Lavagno A, Scarfone AM and Narayana Swamy P 2006 Eur. Phys. J. B 50351

[12] Lavagno A, Scarfone AM and Narayana Swamy P 2005 Rep. Math. Phys. 55 423; 2006 Eur. Phys. J. C 47253

[13] Fetter A and Walecka J 1971 Quantum Theory of many particle systems (McGraw-Hill Book Company)

[14] Landau L and Lifshitz E 1980 Statistical Physics Vol 5 (Pergamon Press, New York)

[15] Parthasarathy R and Viswanathan K 1992 preprint IMSc-92/02-57

[16] Zhang J-z and Osland P 2001 Eur. Phys. J. C 20393

[17] Chaichian M, Gonzalez Felipe R and Montonen C 1993 J. Phys. A: Math. Gen. 264017

[18] Scarfone AM and Narayana Swamy P 2007 Int. J. Mod. Phys. A 226169

[19] Kulish P and Damaskinsky E 1990 J. Phys. A: Math. Gen. 23 L415

[20] Narayana Swamy P 2001 Mod. Phys. Lett. B 15915

[21] Pathria RK 1972 Statistical Mechanics (Pergamon Press, Oxford)

[22] Jackson FH 1909 Am. J. Math. 3826

[23] Finkelstein R 1998 Int. J. Mod. Phys. A 131795

[24] Finkelstein R and Marcus E 1995 J. Math. Phys. 362652

[25] Dobrogowska A and Odzijewicz A 2007 J. Phys. A: Math. Theor. 402023

[26] Dayi ÖF and Duru IH 1997 Int. J. Mod. Phys. A 122373

[27] Minahan JA 1990 Mod. Phys. Lett. A 52625 
[28] Fichtmüller M, Lorek A and Wess J 1996 Z. Phys. C 71533

[29] Cerchiai BL, Hinterding R, Madore J and Wess J 1999 Eur. Phys. J. C 8547

[30] Bohm D 1952 Phys. Rev. 85 166; ibis 1952180

[31] Madelung E 1962 Z. Phys. 40332

[32] Kaniadkis G, Quarati P and Scarfone AM 2002 Physica A 30576

[33] Wang QA 2002 Eur. Phys. J. B 26036142

[34] Bíró TS and Kaniadakis G 2006 Eur. J. Phys. B 503

[35] Bíró TS and Purcsel G 2008 Phys. Lett. A 3721174 